\title{
Factors influencing the usage of different types of malaria prevention methods during pregnancy in Kenya
}

\author{
Shakira Choonara ${ }^{1,2}$, Clifford Obby Odimegwu², Bob Charlestine Elwange $e^{2,3}$
}

1. Centre for Health Policy/MRC Health Policy Research Group; School of Public

Health; Faculty of Health Sciences, University of the Witwatersrand, Johannesburg, South Africa.

2. Demography and Population Studies Programme, Schools of Public Health and Social Sciences,

University of the Witwatersrand, Johannesburg, South Africa.

3. Department of Sociology and Social Administration, Kyambogo University, Uganda.

\begin{abstract}
Background: In sub-Saharan Africa, malaria is a leading cause of morbidity and mortality, which, during pregnancy, is associated with adverse health outcomes for both mother and foetus. Utilization of Insecticide Treated Nets (ITNs) and Intermittent Preventive Therapy (IPTp) is advocated to prevent malaria during pregnancy.

Objective: To examine factors which influence the use of different types of malaria prevention methods among pregnant women in Kenya.

Methods: This study used 2008-09 Kenya Demographic and Health survey. Pregnant women aged 15-49 years were included (622 women). Distribution of the study population was assessed in frequency tables. Bivariate and multivariate logistic regression analysis was employed.

Results: Fifty-two percent of women used ITNs and 38.5\% reported uptake of IPTp. In multivariate analysis age, malaria risk areas, religion, education and income influenced ITN usage, whereas only age, malaria risk areas and marital status were found to influence IPTP uptake.

Conclusions: ITN use and IPTp uptake were well below the 80\% Kenya Malaria Strategy 2006 target. In an effort to increase uptake it is vital for future research to understand reasons for low usage and uptake of malaria prevention programmes so as to enable policy-makers to make informed decisions.
\end{abstract}

Keywords: Malaria prevention methods, Pregnancy, Kenya

DOI: http://dx.doi.org/10.4314/ahs.v15i2.14

\section{Introduction}

Despite efforts to reduce malaria among pregnant women an estimated 30 million pregnant women are still at risk of malaria infection globally ${ }^{1}$. In Africa, the annual rate of malaria related maternal deaths is approximated at 10,000 and 20,000 infant deaths. ${ }^{2}$ Malaria

\section{Corresponding author: \\ Shakira Choonara \\ Centre for Health Policy/MRC \\ Health Policy Research Group; \\ School of Public Health; Faculty of \\ Health Sciences, University of the Witwatersrand, Johannesburg, South Africa. \\ Email: Shakira.Choonara@wits.ac.za}

during pregnancy causes maternal anaemia resulting in low birth weight that predisposes infants to high risk of death. ${ }^{3,1,4}$ Within Kenya an estimated 24 million people are at risk of malaria. Over $30.0 \%$ of hospital admissions are malaria related and the risk is higher among pregnant women, over $69.0 \%$ of which suffer from anaemia. $5,6,7$

The World Health Organisation (WHO) recommends the use of insecticide treated nets (ITNs) and intermittent preventive treatment (IPTp) to prevent malaria during pregnancy. ${ }^{8}$ In Africa, the revised Abuja target was to provide $80.0 \%$ of pregnant women in endemic areas with ITNs and IPTp by 2005. . These measures were taken in order to avert the risk of mortality, anaemia among pregnant women and low birth weight. For instance, in Nigeria there were higher rates of malaria infection among pregnant women who did not make use of an ITN. ${ }^{14}$ The highly protective effect of ITNs 
against malaria was established in Uganda as well. ${ }^{15}$ De- Study variables

spite these malaria prevention benefits, only $50.0 \%$ of The two main outcomes included ITN use and IPTP the women in sub-Saharan Africa use ITNs and 44.0\% have received IPTp. ${ }^{11,12}$

Aligned to the Abuja target, Kenya's national malaria strategy plan of 2001-2010 aimed to ensure that $80 \%$ of pregnant women in the country received ITNs and IPTp by $2010 .{ }^{10}$ As in other parts of Sub-Saharan Africa, adopting malaria prevention has averted the risk of malaria among pregnant women. For example, lower rates of placental malaria were observed among pregnant women who received IPTp treatment in an area of intense malaria transmission in Western Kenya. ${ }^{16}$ However, despite the efforts and their benefits, Kenya has not achieved one of its key goals to combat malaria among pregnant women despite the resources which have been availed. ${ }^{11}$ Less than $50.0 \%$ and $40 \%$ of the population use ITN and IPTp respectively. ${ }^{11,12}$. Despite several studies that have been done in Kenya, most have focused on the effect of malaria on mortality and utilisation of prevention measures. For example, malaria prevention was linked to the decline of low birth weight and mortality prevention among children in an area of high perennial malaria transmission in western Kenya. ${ }^{29,30}$ These studies did not, however, focus on the determinants of malaria prevention. This study therefore attempts to bridge the existing gap. It is relevant because malaria is responsible for $20 \%$ of under-five mortality in Kenya, which threatens annually the lives of 20 million pregnant women and their infants in the country. ${ }^{13}$

\section{Materials and methods}

Data source, study design and participants

This study draws its data from the Kenya Demographic and Health Survey (KDHS) of 2008-2009. It uses the female recode that has got the variables necessary for analysis. Such information includes: pregnancy status, malaria prevention measures, socioeconomic status of the women and the demographic variables. The KDHS is a nationally representative survey; it applies a cross sectional study design with a two-staged stratified sampling with a sample size of 8098 women within the reproductive age. A total of 622 currently pregnant women were included in this cross-sectional study. The power of the sample size was greater than 80 percent, which indicated that the sample was adequate to meet the objectives of the study. uptake. The ITN use variable was created from the 'type of bednet slept under last night' variable in the KDHS A binary variable was created to limit the variable to 'no' (no bednet used) and 'yes', which only treated nets 'nsed (ITNs). The IPTp uptake variable was created from the 'during pregnancy took fansidar for malaria' variable in the KDHS. The variable was limited to eithe those who received IPTp (yes) or who did not receive IPTp during pregnancy (no). These were the only two possible variables which could be adapted to the study from KDHS, which outlined the usage of these measures during pregnancy.

Independent variables assessed for associations with uptake of the prevention methods included demographic variables such as age, malaria risk areas, religion and marital status. Age was categorised as 15-24, 25-34 and above 35 years. Different regions in Kenya were classified according to characteristics of malaria transmission: endemic ( $20 \%$ or higher malaria risk); seasonal endemic (below 5\% risk); or low risk (below $0.1 \%$ risk). Religion was grouped as Christian, Muslim and other, and marital status into never married and married. Measures of socioeconomic status were the leve of education and a wealth index. Five categories (poorest, poor, middle, rich, richest) of the original wealth index variable were reduced to three (poor, middle and wealthiest). The variable assessing a woman's contro over healthcare decisions measures both access to services and women's empowerment, with the categories: respondent and husband/partner; and husband/ partner alone.

\section{Statistical analysis} USA) was used in data analysis. Statistical analysis was done at three levels, namely univariate, bivariate, and multivariate. Univariate analysis generated the frequency distributions to reflect the usage of prevention measures and the background characteristics of the women. The binary logistic regression model was used to analyse the data. The response variables of the study were binary, which led to the selection of this model. It assumes that the observations are a random sample from a population. The model is denoted below: $y=a+\beta_{0}+\beta_{1} X_{1}+\beta_{2} X_{2}+\beta_{3} X_{3} \ldots \beta_{n} X_{n}+\varepsilon_{i}$
Stata 12.0 (Stata Corporation, College Station, TX,
Where: y represents the dichotomous dependent variable which is either a woman used ITN or not, used IPTp or otherwise, $\alpha$ is the gradient, $\beta$ s are the coefficients, $\mathrm{x} . . . \mathrm{xn}$ are the independent variables and $\varepsilon \mathrm{i}$ is the error term with confident level of $95 \%$ and $5 \%$ allowance of error.

The $\beta$ s are the coefficients indicating the odds of a pregnant woman using the insecticide treated nets and the uptake of IPTp given that the person would not use them. In other words, the coefficients reflected the probability that a pregnant woman used the malaria prevention measures. The value of the coefficient ( $\beta$ ) changes as an additional independent variable is added in to the model. The Hosmer and Lemeshow method was used to test the overall goodness of fit of all multivariate logistic regression models.

Odds ratios are vielded through logistic regression models to measure association between independent and dependent variables. Unadjusted odds ratios were yielded through logistic regression. The use of multivariate logistic regression yielded adjusted odds ratio

to test associations between the independent variables the use of different types of malaria prevention methods during pregnancy, and also controlled for potential confounding factors. A combination of forward and backward elimination methods were used to create the final logistic regression models.

\section{Results}

Malaria prevention and background characteristics of the respondents

Table 1 shows that approximately half of the pregnant women used ITNs $(52.9 \%)$, while only $24.1 \%$ received IPTp. Forty-seven percent of the study sample was aged $15-24$ years, and $12.9 \%$ older than 35 years. Seventy-five percent of the sample was Christian and $21.4 \%$ were Muslim. Other characteristics of participants indicate that close to $90.0 \%$ of participants were married. For $56.3 \%$ of women, primary education was the highest level reached. A low percentage of pregnant women $(22.8 \%)$ reported higher education. Most women made healthcare decisions together with their partners, though for a quarter of women still had their health care decisions made solely by their partners.

Table 1: Outcomes \& Characteristics of Currently Pregnant Women in Kenya 2008-2009

\begin{tabular}{|c|c|c|c|}
\hline Var. group & Variable & Total Population $(\mathrm{N}=622)$ & Percentage (\%) \\
\hline \multirow{2}{*}{ 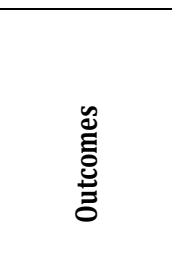 } & \begin{tabular}{|l} 
ITN usage \\
No \\
Yes
\end{tabular} & $\begin{array}{l}293 \\
329\end{array}$ & $\begin{array}{l}47.1 \\
52.9\end{array}$ \\
\hline & \begin{tabular}{|l}
$\mid \begin{array}{l}\mid \text { IPTp Uptake } \\
\text { No } \\
\text { Yes }\end{array}$ \\
\end{tabular} & $\begin{array}{l}472 \\
150\end{array}$ & $\begin{array}{l}75.8 \\
24.1\end{array}$ \\
\hline \multirow{3}{*}{ 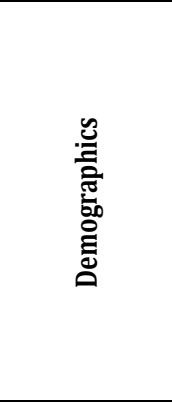 } & \begin{tabular}{|l|} 
Age \\
$15-24$ \\
$25-34$ \\
$35+$ \\
$35+$
\end{tabular} & $\begin{array}{r}295 \\
247 \\
80\end{array}$ & $\begin{array}{l}47.4 \\
39.7 \\
12.9\end{array}$ \\
\hline & \begin{tabular}{|l|} 
Religion \\
Christian \\
Muslim \\
Other
\end{tabular} & $\begin{array}{c} \\
466 \\
133 \\
23\end{array}$ & $\begin{array}{c} \\
75.0 \\
21.4 \\
3.6\end{array}$ \\
\hline & \begin{tabular}{|l|} 
Marital status \\
Never married \\
Currently married \\
\end{tabular} & $\begin{array}{r}67 \\
555 \\
\end{array}$ & $\begin{array}{l}10.8 \\
89.2 \\
\end{array}$ \\
\hline \multirow{2}{*}{ 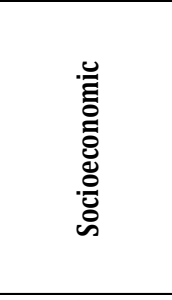 } & \begin{tabular}{|l|} 
Level of education \\
No education \\
Primary \\
Higher Education
\end{tabular} & $\begin{array}{l}130 \\
350 \\
142 \\
\end{array}$ & $\begin{array}{l}20.9 \\
56.3 \\
22.8\end{array}$ \\
\hline & \begin{tabular}{|l} 
Wealth index \\
Poor \\
Middle \\
Wealthiest
\end{tabular} & $\begin{array}{l}275 \\
106 \\
241\end{array}$ & $\begin{array}{l}44.2 \\
17.0 \\
38.8\end{array}$ \\
\hline \multirow[t]{2}{*}{ 产 } & $\begin{array}{l}\text { Health decisions } \\
\text { Respondent \& husband/partner }\end{array}$ & 398 & 64.1 \\
\hline & Husband/partner alone & 224 & 36.0 \\
\hline
\end{tabular}

African Health Sciences Vol 15 Issue 2, June 2015 
Determinants influencing use of different types of to two times more likely to utilise ITNs (AOR=1.52 malaria prevention measures the uptake of prevention methods. Age was associat- and $83.0 \%$ less likely to use ITNs than women in ened with both outcomes. Women aged 25-34 were close demic areas ( $\mathrm{AOR}=0.58 ; 95 \% \quad \mathrm{CI}=0.39-0.87$ versus

$\mathrm{AOR}=0.17 ; 95 \% \mathrm{CI} 0.10-0.30)$.

\section{Table 2 Factors influencing use of different types of malaria prevention methods among pregnant women in Kenya 2008-09}

\begin{tabular}{|c|c|c|c|c|}
\hline \multirow{2}{*}{ Variables } & \multicolumn{2}{|c|}{ ITN usage } & \multicolumn{2}{|c|}{ IPTp administration } \\
\hline & Unadjusted Odds Ratios & Adjusted Odd Ratios & Unadjusted Odds Ratios & Adjusted Odds Ratios \\
\hline $\begin{array}{l}\text { Age } \\
15-24 \\
25-34 \\
35+\end{array}$ & 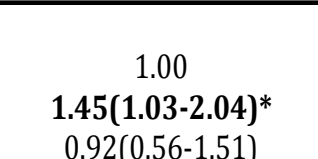 & $\begin{array}{c}1.00 \\
\mathbf{1 . 5 2 ( 1 . 0 4 - 2 . 2 1 )})^{*} \\
1.2(0.65-1.91)\end{array}$ & $\begin{array}{c}1.00 \\
1.79(1.20-2.67)^{*} \\
1.52(0.85-2.07)\end{array}$ & $\begin{array}{c}1.00 \\
\mathbf{1 . 8 3 ( 1 . 2 1 - 2 . 8 1 ) ^ { * }} \\
1.52(0.83-2.79)\end{array}$ \\
\hline $\begin{array}{l}\text { Malaria risk areas } \\
\text { Endemic } \\
\text { Seasonal risk } \\
\text { Low Risk } \\
\end{array}$ & 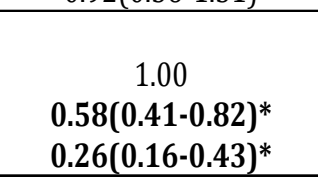 & 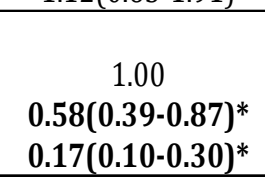 & $\begin{array}{c}1.00 \\
0.68(0.46-1.01) \\
\mathbf{0 . 2 8 ( 0 . 1 4 - 0 . 5 6 ) *}\end{array}$ & 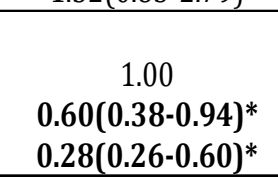 \\
\hline $\begin{array}{l}\text { Religion } \\
\text { Christian } \\
\text { Muslim } \\
\text { Other }\end{array}$ & $\begin{array}{c}1.00 \\
1.75(1.18-2.62)^{*} \\
0.45(0.18-1.13)\end{array}$ & $\begin{array}{c}1.00 \\
2.26(1.35-3.76)^{*} \\
0.60(0.23-1.59)\end{array}$ & $\begin{array}{c}1.00 \\
1.29(0.84-2.00) \\
0.32(0.07-1.40)\end{array}$ & $\begin{array}{c}1.00 \\
1.21(0.72-2.06) \\
0.28(0.06-1.28)\end{array}$ \\
\hline $\begin{array}{l}\text { Marital status } \\
\text { Never married } \\
\text { Currently married }\end{array}$ & $\begin{array}{c}1.00 \\
2.53(1.48-4.33)^{*}\end{array}$ & $\begin{array}{c}1.00 \\
2.29(1.28-4.07)^{*}\end{array}$ & $\begin{array}{c}1.00 \\
\mathbf{2 . 5 4 ( 1 . 1 8 - 5 . 4 4 )}\end{array}$ & $\begin{array}{c}1.00 \\
\text { 2.19(0.99-4.82)* }\end{array}$ \\
\hline $\begin{array}{l}\text { Level of education } \\
\text { No education } \\
\text { Primary } \\
\text { Higher Education }\end{array}$ & $\begin{array}{c}1.00 \\
1.18(0.79-1.78) \\
\mathbf{1 . 6 4 ( 1 . 0 1 - 2 . 6 4 )} \\
\end{array}$ & $\begin{array}{c}1.00 \\
1.36(0.78-2.38) \\
\mathbf{2 . 0 7 ( 1 . 0 7 - 4 . 0 0})^{*} \\
\end{array}$ & $\begin{array}{c}1.00 \\
0.97(0.61-1.54) \\
0.79(0.44-1.38) \\
\end{array}$ & $\begin{array}{c}1.00 \\
0.95(0.51-1.75) \\
0.89(0.43-1.85) \\
\end{array}$ \\
\hline $\begin{array}{l}\text { Wealth index } \\
\text { Poor } \\
\text { Middle } \\
\text { Wealthiest }\end{array}$ & $\begin{array}{c}1.00 \\
1.12(0.71-1.75) \\
1.11010-200)\end{array}$ & $\begin{array}{c}1.00 \\
1.27(0.77-2.09) \\
\mathbf{1 . 8 3 ( 1 1 7 - 2 . 8 3})^{*}\end{array}$ & $\begin{array}{c}1.00 \\
0.87(0.52-1.45) \\
0.68(0.5-1.03)\end{array}$ & $\begin{array}{c}1.00 \\
0.93(0.53-1.63) \\
0.82(0.51-1.32)\end{array}$ \\
\hline $\begin{array}{l}\text { Health decisions } \\
\text { Respondent and husband/partner } \\
\text { Husband/partner alone }\end{array}$ & $\begin{array}{c}1.00 \\
1.07(0.76-1.53)\end{array}$ & - & $\begin{array}{c}1.00 \\
1.00(0.66-1.47)\end{array}$ & (2) \\
\hline
\end{tabular}

In contrast to endemic areas, fewer pregnant women in seasonal and low risk areas received IPTp $(\mathrm{AOR}=$ 0.60 ; $95 \%$ CI $0.38-0.94$ versus $\mathrm{AOR}=0.28$; $95 \% \mathrm{CI}$ $0.26-0.60)$. Muslim women were 2.3 times more likely to use ITNs in comparison to Christian women $(95 \% \mathrm{CI}$ $\mathrm{AOR}=1.35-3.76)$. Married women were higher users of ITNs than never-married ones as reflected in the odd ratios $(\mathrm{AOR}=2.29 ; 95 \% \mathrm{CI}=1.28-4.07)$. Similarly, relative to never-married women, the married ones were more likely to receive IPTp (AOR=2.19; 95\% CI $=0.99$ 4.82) women from poorer households (AOR=1.83; $95 \%$ $\mathrm{CI}=1.17-2.83)$.
The higher uptake of malaria prevention among women In sub-Saharan Africa the effectiveness of ITNs and from wealthier households was also noted in previous IPTp during pregnancy is well established. In Burkina studies. In Uganda, for example, high costs of ITNs Faso it was found that an increased dose of IPTp dur- inhibited malaria prevention among pregnant women. ${ }^{26}$ ing pregnancy was associated with a reduced risk of low In this study, significant associations were established birth weight of infants in Burkina Faso. ${ }^{17}$ The utilisa- between wealth index and the use of ITNs. A possible tion of ITNs among pregnant women in Malawi was explanation for the low usage of ITNs and IPTp among low birth weight of infant ${ }^{8}$ It has been agued in previous literature that there is a need to understand factors which influence the use of ITNs and IPTp. ${ }^{18}$ This study examined the effects of several demographic, socioeconomic and empowerment determinants influencing the use of different types malaria prevention measures during pregnancy.

A prior study indicates no association between marital status age, and ITN use in Ethiopia. ${ }^{19}$ In Kenya associations were detected between age, marital status and the outcome measures of this study (ITN, and IPTp). Residing in different malaria risk areas was linked with use of ITNs, IPTp and combined uptake. Levels of uptake during pregnancy were lower in parts of Kenya with less malaria transmission, as found in a study in Ghana. ${ }^{20}$ Qualitative research in Ethiopia showed that when women perceive the risk of malaria to be low, malaria ceases to be a serious problem psychologically, resulting in low usage of preventive measures. ${ }^{21}$ Similar to findings in Nigeria, religion played an important role in the uptake of malaria prevention measures. ${ }^{22}$ This study merely displayed association although further insight into examining the impact of religion on use of malaria prevention measures is clearly needed.

Some associations were established between socioeconomic variables and the use of ITNs. Once these were adjusted, no associations were made between these variables and IPTp administration increased odds of the usage of ITNs, and increased with education support findings in Kenya where there was an association between the level of knowledge of malaria and education. ${ }^{23}$ It is also argued that educated women tend to have more knowledge regarding malaria and are therefore found to make greater use of these measures. ${ }^{24}$ These results are similar to an earlier study where significant associations were established between women with a primary level of education and IPTp administration in Uganda. ${ }^{25}$

households and ITN use were considerably higher than preonant Kenyan women could be due to the cost associated with these measures. This is supported by the findings in Kilifi district in western Kenya where close to $85 \%$ of women mentioned lack of money as the major reason for the non-usage of ITNs during pregnan$\mathrm{cy}^{23}$ Other direct and indirect costs may include long queues at antenatal clinics, limited resources for IPTp a health facilities and transport costs to the clinics.

\section{Study Limitations}

The study has a few limitations although these are not strong enough to invalidate the results. The limitations include the following:

1) The different recall periods used in the measure of IPTp and ITN use in the KDHS survey limits the validity of the combined uptake variable. Data collected as part of the KDHS was based on individual responses from women which are subject to recall bias. pregnancy status and the time for initiating the malaria prevention measures. It is possible that women might have just confirmed their pregnancy status close to the time of data collection and may not have actually use any prevention methods as there is a time lag between realising pregnancy status and enrolling for antenatal care services. Such scenarios could have led to a possible underestimation of the use of prevention methods. 3) The study only relied on the quantitative approach. that most of the women failed to take malaria prevention. This would lead to a deeper understanding of the causes that could be used in policy designs and implementation by health officials, administrators and the community leaders. ${ }^{14}$

\section{Conclusions}

The levels of usage of ITNs $(52.9 \%)$ and IPTp uptake $24.1 \%$ ) are well below the $80 \%$ Kenva Malaria Strategy 2006 target, which concerns the adverse effects of malaria during pregnancy on both mothers and infants. A range of socio-demographic variables was found to influence use of different types of malaria prevention
2) The KDHS did not capture the period between It would have been important to establish the reasons 
measures during pregnancy in Kenya. In particular, low Death in the Extremely Low Birth Weight in Infant. levels of use was recorded among women in season- Pediatrics. 1999; 2(133).

al and low risk areas, less educated women, those who 5. O'Meara, WP, Mangeni JN, Steketee R, Greenwood were not married, women of different religions, and B. Changes in the burden of malaria in sub-Saharan Afthose from poor and middle-income households. The rica. LANCET INFECT DIS. 2010; 10: 545-55. findings of this study indicate that women in these cat- 6.KNBS: ICF Macro: Kenya Demographic and Health egories in Kenya be specifically targeted to optimise the Survey 2008-09. Calverton, Maryland. 2010. uptake of prevention measures during pregnancy. While 7. Ouma P, Eijk AM, Hamel MJ, Parise M, Ayisi J.G, associations have been detected between these variables Otieno K. Malaria and anaemia among pregnant womand the actual usage of these measures, this study fails en at first antenatal clinic visit in Kisumu, Western Kento provide depth and insight as to why or how these ya. Trop Med Int Health. 2007; 12(12): 1515-1523. factors are associated with use of these measures dur- 8. Feng G, Simpson JA, Chaluluka E, Molyneux ME, ing pregnancy. More studies need to be geared towards Rogerson SJ. Decreasing burden of malaria in pregnanunderstanding the influence of these factors in Kenya cy in Malawian women and its relationship to use of as well as in other settings, due to there being minimal intermittent preventative therapy or bed nets. PLoS One. literature in this regard. Furthermore, there is a need 2010; 5(8): 1-9.

for these studies to be coupled with qualitative research 9. Beer N, Ali AS, Savigny D, Al-Maf A, Ramsan M, in order to have a thorough understanding of how and Abass A et al. System Effectiveness of a Targeted Free why these factor thuly infuence the use of $p$ measures.

\section{Acknowledgements}

This paper is an extract of a broader Master's degre completed in Demography and Population studies at the University of the Witwatersrand, South Africa. SC received financial support from South Africa's National Research Foundation (NRF) through the 2012 Freestanding Masters scholarship. Gratitude is extended to the William and Flora Hewlett Foundation, USA for granting support through the interdisciplinary Demography and Population Studies programme of the School of Social Sciences and Public Health, University of the Witwatersrand. The funders did not, however, influence the activities of the study. The authors would also like to thank Measure DHS for providing datasets.

\section{References}

1. Guyatt, H.L., Snow, R,W. Impact of malaria during pregnancy on low birth weight in Sub-Saharan Africa. CLIN MICROBIOL REV. 2004; 17(4): 760-769.

2. Okpere E, Enabudoso E, Osemwenkha A. Malaria in pregnancy. Niger Med J, 2010.

3. Kabanywanyi AM, MacArthur JR, Stolk WA, Habbema JDF, Mshinda $\mathrm{H}$, Bloland $\mathrm{P}$ et al. Malaria in pregnant women in an area with sustained high coverage of insecticide treated bed nets. MALARLA J. 2008;7(133): 1-8.

4. Barton L, Joan E, Pavlova H, Pavlova, Z. Causes of Mass Distribution of Long Lasting Insecticide Treated Nets in Zanzibar, Tanzania. Malaria J. 2010;9(173): 1-10 10. Division of Malaria Control. Kenya National Malar ia Strategy 2001-2010.

11. World Malaria Report 2011. World Health Organisation.

12. Ejik A, Blockland I, Slutsker L, Odhiambo F, Ayisi , Bles, $\mathrm{H}$ et al. Use of Intermittent Preventative Treatment for malaria in pregnancy in rural area of Western Kenya with high coverage of insecticide treated bed nets. Am J Trop Med Hyg. 2005;10(11): 1134-114.

13. Scott JAG, Berkley JA, Mngwangi I, Ochola L, Uyoga S, Macharia A et al. Relation between falciparum malaria and bacteraemia in Kenyan children: a population-based, case-control study and a longitudinal study. Lancet. 2011; 378: 1316-23.

14. Houmsou R, Amuta E, Sar T. Malaria prevention during pregnancy: awareness and factors contributing to disease occurrence among pregnant women in Gboko Metropolis, Benue State Nigeria. JHST. 2010; 36-40.

15. Namusoke F, Rasti N, Kironde F, Matswahlgren M, Mirembe F. Malaria burden in pregnancy at Mulago National Referral Hospital in Kampala, Uganda. Malaria Res Treat: 1-11

6. Parise M, Ayisi J, Nahlen B, Schultz L, Roberts J, Misore A et al. Efficacy of sulphadoxine-pyrimethamine for prevention of placental malaria in an area with high prevalence of malaria and Human Immunodefeciency Virus Infection. Am J Trop Med Hyg. 1998; 59(5): 813-882.
17. Sirmia A, Cotte A, Konate A, Moran A, Asamoa K, Bougouma $\mathrm{E}$ et al. Malaria prevention during pregnancy: assessing the disease burden one year after implementing a program of Intermittent Preventative Treatment in Koupela District, Burkina Faso. Am J Trop Med Hyg, 2006, 75(2): 205-211.

18. Nuwaha F. Factors influencing the use of bednets in Mbaraa municipality of Uganda. Am I Trop Med Hyg. 2001; 65(6): 877-872.

19. Belay M, Deressa W. Use of insecticide treate hets by pregnant women and associated factors in pre-dominantly rural population in Northern Ethiopia. Trop Med Int Health. 2008; 13(10): 1303-1313.

20. Cruz N, Crookston B, Dearden K, Gray B, Ivin $\mathrm{N}$, Alder $\mathrm{S}$, et al. Who sleeps under nets in Ghana? A doer/non-doer analysis of malaria prevention behaviours. Malaria J. 2006; 5(61): 1-10.

21. Baume C, Reithinger R, Woldehanna S. Factors associated with the use/non-use of mosquito nets owned in Oramia and Amhara regional states, Ethiopia. Malar ia J. 2009; 8(264): 1-11.
22. Haslegrave M, Bernstein, S. ICPD goals: essential to millennium development goals. Reprod Health Matter. 2005;13(25): 106-108.

23. Njoroge FK, Kimani VN, Ongore D, Akwale WS Use of insecticide treated bed nets among pregnant women in Kilifi District, Kenya. E Afr Med J. 2009. 6(7): 314-322.

24. Napoleon RP, Anyangu AS, Omolocan J, Ongus JR. Preventing malaria during pregnancy: factors determining the use of insecticide treated bed nets and intermittent preventive therapy in Juba. SSMJ. 2011; 4(2): $1-44$

25. Sangare L, Stergachis A, Brentlinger P, Richardson B. Kiwuwa M, Wiess N. Determinants of the use of intermittent preventive treatment of malaria in pregnancy: Jinja, Uganda. PLoS One. 2010; 5(11): 1-7. 26. Mbonye A, Neema S, Magnussen P. (2003). Preventing malaria in preonancy: a study of perceptions and 